\title{
Stabilization of a 1-D tank modeled by the shallow water equations
}

\author{
Christophe Prieur Jonathan de Halleux
}

\begin{abstract}
We consider a tank containing a fluid. The tank is subjected to a onedimensional horizontal move and the motion of the fluid is described by the shallow water equations. By means of a Lyapunov approach, we deduce control laws to stabilize the fluid's state and the tank's position. Although global asymptotic stability is yet to be proved, we numerically simulate the system and observe the stabilization for different control situations.
\end{abstract}

\section{Introduction}

We consider an 1-D tank containing an inviscid incompressible irrotational fluid. We are interested in the stabilization problem of the fluid state (height and speed relative to the tank) and the tracking problem of the trajectory of the tank (position, speed and acceleration) to a prescribed trajectory (e.g. a prescribed final position of the tank).

We suppose that the horizontal acceleration is small compared to the gravity constant and that the height of the fluid is small compared to the length of the tank. Hence we describe the dynamic of the fluid by the shallow water equations (see [3, Section 4.2] and [14] and references therein).

The acceleration defines the control variable. We exhibit a stabilizing feedback based on a Lyapunov approach (see Theorem 3). We emphasize that we proceed by increasing the complexity of the Lyapunov function. First we stabilize only the fluid's state (Section 3.1), then we stabilize also the tank's speed (Section 3.2) and then, we use a forward approach (see [10]) to stabilize the entire state of the system fluid-tank in Theorem 3.

Many physical motivations can be found in $[6,12]$ for looking such a feedback stabilizing the entire state of the system fluid-tank. Some results can be found in [11] concerning the problem of the stabilization of a tank, but the input is defined

Keywords : Quasilinear hyperbolic PDEs, shallow water equations, Lyapunov approach, boundary control, numerical resolution.. 
as a flexible or a rigid wave generators and the equations are linearized around the equilibrium. Here we choose a different model of the control system.

The asymptotic stability is yet to be proved but we check numerically that the result is attained (see Section 4.1).

Note that the shallow water equations, linearized around a suitable equilibrium, are uncontrollable (see [7]) but the non-linear shallow water equations are locally controllable around the equilibriums (see [1]). We check numerically that the stabilization property is achieved with the non-linear terms of the shallow water equations in Section 4.2.

\section{Model description}

The shallow water equations describe the motion of a perfect fluid under gravity $g$ with a free boundary:

$$
\begin{aligned}
& \frac{\partial H}{\partial t}(t, x)+\frac{\partial}{\partial x}(H V)(t, x)=0, \\
& \frac{\partial V}{\partial t}(t, x)+\frac{\partial}{\partial x}\left(g H+\frac{V^{2}}{2}\right)(t, x)=-\ddot{D}(t),
\end{aligned}
$$

where $x \in[0, L]$ is the spatial coordinate attached to the tank, $t \in[0, T]$ is the time coordinate, $T>0, H(t, x)$ denotes the height of the liquid, $V(t, x)$ denotes the horizontal speed of the fluid in the referential attached to the tank, $D$ is the position of the tank in the world coordinates, $\dot{D}$ and $\ddot{D}$ are respectively the first and second derivative of $D$ with respect to the time $t$. See Figure 1 . Note that the shallow water equations can been dericated from the Euler equations for the perfect irrotational and incompressible fluids (see [4]).

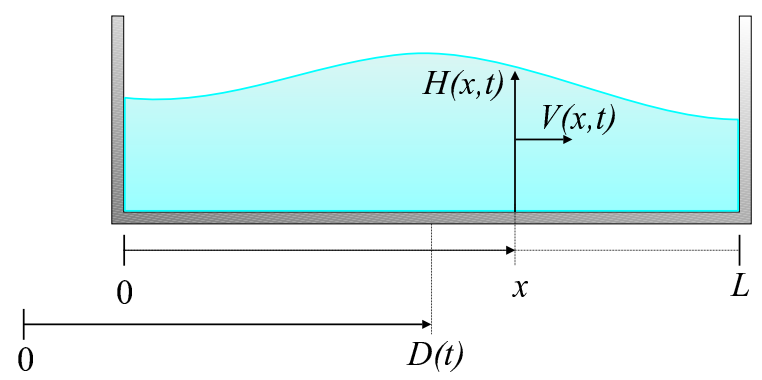

Figure 1: A tank of length L containing a fluid.

The boundary conditions are given by, for all $t$ in $[0, L]$,

$$
V(t, 0)=0 \quad, \quad V(t, L)=0
$$

Let us denote $\bar{H}(x)$ and $\bar{V}(x)$ the steady state values of $(H, V)$ along the reach, i.e.:

$$
\frac{\partial}{\partial x}(\bar{H} \bar{V})=0, \frac{\partial}{\partial x}\left(g \bar{H}+\frac{\bar{V}^{2}}{2}\right)=-\bar{A},
$$


where $\bar{A}$ is a constant number defining the constant acceleration of the tank. The above equations (4) can be rewritten as follows

$$
\forall x \in[0, L], \quad \begin{aligned}
& \bar{V}(x)=0, \\
& \bar{H}(x)=\bar{H}\left(\frac{L}{2}\right)-\left(x-\frac{L}{2}\right) \frac{\bar{A}}{g} .
\end{aligned}
$$

In fact, we can compute the (constant) volume of liquid in the tank:

$$
\mathrm{Vol}=\int_{0}^{L} \bar{H} d x=L \bar{H}\left(\frac{L}{2}\right) .
$$

We define our control variable $u$

$$
u=\ddot{D}-\bar{A} .
$$

Let $|$.$| be a norm of \mathbb{R}$ and $|.|_{1}$ be the norm on $\mathcal{C}^{1}([0, L])$ defined by, for all $f$ in $\mathcal{C}^{1}([0, L])$,

$$
|f|_{1}=\max _{x \in[0, L]}|f(x)|+\max _{x \in[0, L]}\left|f^{\prime}(x)\right|,
$$

where $/$ denotes the partial derivative with respect to $x$.

Given an initial condition $(\widetilde{H}, \widetilde{V})$ for the fluid and an initial acceleration of the $\operatorname{tank} \widetilde{A}$, note that there exist sufficient conditions for the existence of a solution of the Cauchy problem (1), (2) and (3) (see [8, Theorem 4.2, page 96]):

Claim 2.1 There exists a strictly positive constant $\varepsilon$ such that, for any $(\widetilde{H}, \widetilde{V})$ in $C^{1}([0, L])^{2}$ satisfying the compatibility conditions:

$$
\begin{aligned}
2 g \widetilde{H}(0) \widetilde{H}^{\prime}(0)+\widetilde{V}(0) \widetilde{V}^{\prime}(0) & =-\widetilde{A}, \\
2 g \widetilde{H}(L) \widetilde{H}^{\prime}(L)+\widetilde{V}(L) \widetilde{V}^{\prime}(L) & =-\widetilde{A},
\end{aligned}
$$

and

$$
|\widetilde{H}-\bar{H}|_{1}+|\widetilde{V}-\bar{V}|_{1}<\varepsilon,
$$

the hyperbolic system (1) and (2) with initial conditions:

$$
H(0, x)=\widetilde{H}(x), \quad V(0, x)=\widetilde{V}(x) \quad, \forall x \in[0, L],
$$

and with boundary conditions (3) has one and only one solution of class $C^{1}$ defined on $[0, L] \times[0, T)$, for some $T>0$.

Now let us define $\mathcal{E}=\{(H, V, D, S)\}$ the affine subspace of $\mathcal{C}^{1}([0, L]) \times \mathcal{C}^{1}([0, L]) \times$ $\mathbb{R} \times \mathbb{R}$ such that we have $\mathrm{Vol}=\int_{0}^{L} \widetilde{H}(x) d x$, where Vol is defined by (6), and $H_{x}(0)=H_{x}(L)=-\frac{u+\bar{A}}{g}$.

We are interested in the problem of the local stabilization to the equilibrium $(\bar{H}, \bar{V}, \bar{D}, \bar{S})$ with $\bar{A}$ in $\mathbb{R}$ fixed, satisfying (5) by the control $u$, i.e. we are looking for a function $u:[0,+\infty) \times \mathcal{E} \rightarrow \mathbb{R}$ such that we have the following two properties 1. There exists $C>0$ such that, for all $(\widetilde{H}, \widetilde{V}, \widetilde{D}, \widetilde{S})$ in $\mathcal{E}$ satisfying the conditions (8)-(10) and

$$
|\widetilde{H}-\bar{H}|_{1}+|\widetilde{V}-\bar{V}|_{1}+|\widetilde{D}-\bar{D}|+|\widetilde{S}-\bar{S}| \leq C
$$


there exists one and only one $(H, V, D, S):[0,+\infty) \rightarrow \mathcal{E}$ such that, we have (1)-(3) where, for all $t \geq 0$,

$$
\ddot{D}(t)-\bar{A}=u(t, H(t, .), V(t, .), D(t), S(t)),
$$

such that we have

$$
H(0, .)=\widetilde{H}, V(0, .)=\widetilde{V}, D(0)=\widetilde{D}, S(0)=\widetilde{S},
$$

and, for all $t \geq 0$,

$$
\dot{D}(t)=S(t)
$$

Moreover this function satisfies

$|H(t, .)-\bar{H}|_{1}+|V(t, .)-\bar{V}|_{1}+|\dot{D}(t)-\bar{S}-\bar{A} t|+\left|D(t)-\bar{D}-\bar{S} t-\frac{1}{2} \bar{A} t^{2}\right| \rightarrow_{t \rightarrow+\infty} 0$.

2. For all $\varepsilon>0$, there exists $\eta>0$ such that, if $(\widetilde{H}, \widetilde{V}, \widetilde{D}, \widetilde{S})$ in $\mathcal{E}$ satisfies the conditions (8)-(10) and

$$
|\widetilde{H}-\bar{H}|_{1}+|\widetilde{V}-\bar{V}|_{1}+|\widetilde{D}-\bar{D}|+|\widetilde{S}-\bar{S}| \leq \eta,
$$

if $(H, V, D, S):[0,+\infty) \rightarrow \mathcal{E}$ is such that, (1)-(3), (11)-(13) hold, then we have

$$
|H(t, .)-\bar{H}|_{1}+|V(t, .)-\bar{V}|_{1}+|\dot{D}(t)-\bar{S}-\bar{A} t|+\left|D(t)-\bar{D}-\bar{S} t-\frac{1}{2} \bar{A} t^{2}\right| \leq \varepsilon, \forall t \geq 0
$$

In all the following we are interested in this problem and we propose a Lyapunov control design. Then we check that, numerically, the stabilization is attained.

\section{Lyapunov control design}

We want to build a Lyapunov candidate to stabilize the state of the system fluidtank. The idea of this section is to build a Lyapunov function which is a general tool to prove, for a differential equation, that the origin is an asymptotic stable equilibrium.

\subsection{Stabilization of the fluid's state $(H, V)$}

Let us consider first the stabilization of the fluid's state. We want to find an entropy $E(H, V)$ and an entropic flux $F(H, V)$. There is an infinite number of entropies for the shallow water equations (see [16, Volume II, Section 9.3]), one of them is derived from the moments of the fluid:

$$
\begin{aligned}
& E(H, V)=H \frac{V^{2}}{2}+g \frac{(H-\bar{H})^{2}}{2}, \\
& F(H, V)=H \frac{V^{3}}{2}+g V H(H-\bar{H}) .
\end{aligned}
$$

We can define the Lyapunov candidate (see [2]) $R_{1}:[0,+\infty) \rightarrow \mathbb{R}$

$$
R_{1}(t)=\lambda_{1} \int_{0}^{L} E(H(t, x), V(t, x)) d x,
$$


for a constant $\lambda_{1}>0$ introduced for the tuning of the control. Note that $R_{1}$ is positive and is zero only at the point $(H, V)=(\bar{H}, \bar{V})$. We can now exhibit a class of control laws for $u$, making $R_{1}$ decrease, as stated in the following

Theorem 1 For any positive gain $\lambda_{1}$, the control law

$$
u_{1}(t)=\lambda_{1} \int_{0}^{L}(H V)(t, x) d x
$$

makes $R_{1}$ decrease, i.e. $\dot{R}_{1} \leq 0$. Moreover $\dot{R}_{1}=0$ if $(H, V)=(\bar{H}, \bar{V})$.

Remark 3.1 We can not apply LaSalle's Theorem since we do not know, if the fact that the equality $\dot{R}_{1}(t)=0$ holds for all $t$, yields $(h, v)=(0,0)$. Note moreover that in an infinite dimensional space of functions, we have to prove a suitable compactness property.

Proof. We derive (16) with respect to $t$ :

$$
\dot{R}_{1}=\lambda_{1} \int_{0}^{L}\left(\frac{\partial E}{\partial V} \frac{\partial V}{\partial t}+\frac{\partial E}{\partial H} \frac{\partial H}{\partial t}\right) d x
$$

Hence, using (1), (2) and (14), we have

$$
\dot{R}_{1}=-u_{1} \lambda_{1}\left(\int_{0}^{L} H V d x-[F]_{0}^{L}\right) .
$$

Using the boundary conditions (3) and (15), we have $[F]_{0}^{L}=0$, hence a natural expression for $u_{1}$ is (17).

\subsection{Stabilization of the fluid's state $(H, V)$ and of the tank's speed $\dot{D}$}

In this section we want to stabilize also the tank's speed $\dot{D}$ around $\bar{S}+\bar{A} t$. In order to achieve this, we introduce a modified "kinetic energy" of the tank in (16),

$$
R_{2}(t)=R_{1}(t)+\lambda_{2} \frac{(\dot{D}(t)-\bar{S}-\bar{A} t)^{2}}{2},
$$

where $R_{1}$ is defined by (16) and $\lambda_{2}$ is a positive constant introduced for the tuning of the controller.

Note that $R_{2}$ is positive and is zero only at the point $(H, V, \dot{D})=(\bar{H}, \bar{V}, \bar{S}+\bar{A} t)$.

Using the same approach as before, we can now propose a class of control laws for $u$, making $R_{2}$ decrease, as stated in the following

Theorem 2 For any positive gains $\lambda_{1}, \lambda_{2}$, the control law

$$
u_{2}(t)=\lambda_{1} \int_{0}^{L}(H V)(t, x) d x-\lambda_{2}(\dot{D}(t)-\bar{S}-\bar{A} t),
$$

makes $R_{2}$ decrease, i.e. $\dot{R}_{2} \leq 0$. Moreover $\dot{R}_{2}=0$ if $(H, V, \dot{D})=(\bar{H}, \bar{V}, \bar{S}+\bar{A} t)$. 
Proof. We compute the first derivative of (19) with respect to $t: \dot{R}_{2}=\dot{R}_{1}+\lambda_{2}(\dot{D}-$ $\bar{S}-\bar{A} t)(\ddot{D}-\bar{A})$. Hence, using (18) and (7), we have

$$
\dot{R}_{2}=-u_{2}\left(\lambda_{1} \int_{0}^{L} H V d x-\lambda_{2}(\dot{D}-\bar{S}-\bar{A} t)\right) .
$$

Thus a natural expression for $u_{2}$ is (20).

\subsection{Complete stabilization}

In Section 3.2 we propose a candidate control law to stabilize the state of the fluid and the speed of the tank. In this section we want to stabilize the entire function $D$ and not only its first derivative. To do this we use a forward approach to find a modification of the Lyapunov function $R_{2}$ defined by (19). See e.g. [10]. Thus we have to find a function of the state whose time-derivative is proportional to $\dot{R}_{2}$. This leads to

$R_{3}(t)=R_{2}(t)+\frac{\lambda_{3}}{2}\left(-\lambda_{2}\left(D(t)-\bar{D}-\bar{S} t-\frac{\bar{A} t^{2}}{2}\right)-\lambda_{1} \int_{0}^{L}\left(\int_{0}^{x}(H-\bar{H})(t, \xi) d \xi\right) d x\right)^{2}$

where $\lambda_{1}, \lambda_{2}$ and $\lambda_{3}$ are three positive constants introduced for the tuning of the controller and $R_{2}$ is defined by (19). Note that $R_{3}$ is positive and is zero only at the point $(H, V, \dot{D}, D)=\left(\bar{H}, \bar{V}, \bar{S}+\bar{A} t, \bar{D}+\bar{S} t+\frac{\bar{A} t^{2}}{2}\right)$. We have the following

Theorem 3 For any positive gains $\lambda_{1}, \lambda_{2}$ and $\lambda_{3}$ the control law $u_{3}$

$$
\begin{aligned}
u_{3}= & \lambda_{1} \int_{0}^{L} H V-\lambda_{2}(\dot{D}-\bar{S}-\bar{A} t)-\lambda_{2} \lambda_{3}\left(D(t)-\bar{D}-\bar{S} t-\frac{1}{2} \bar{A} t^{2}\right) \\
& -\lambda_{1} \lambda_{3} \int_{0}^{L}\left(\int_{0}^{x}((H-\bar{H})(t, \xi)) d \xi\right) d x
\end{aligned}
$$

makes $R_{3}$ decrease, i.e. $\dot{R}_{3} \leq 0$. Moreover $\dot{R}_{3}=0$ if $(H, V, \dot{D}, D)=(\bar{H}, \bar{V}, \bar{S}+$ $\left.\bar{A} t, \bar{D}+\bar{S} t+\frac{\bar{A} t^{2}}{2}\right)$.

Proof. Note that due to (1) we have

$$
\begin{aligned}
\frac{d}{d t}\left(\int_{0}^{L}\left(\int_{0}^{x} H-\bar{H}\right) d x\right) & =-\int_{0}^{L}\left(\int_{0}^{x} \frac{\partial(H V)}{\partial x}\right) d x \\
& =-\int_{0}^{L} H V
\end{aligned}
$$

therefore the time-derivative of $R_{3}$ is

$\dot{R}_{3}=\dot{R}_{2}+\lambda_{3}\left(-\lambda_{2}(\dot{D}-\bar{S}-\bar{A} t)+\lambda_{1} \int_{0}^{L} H V\right)\left(-\lambda_{2}\left(D-\bar{D}-\bar{S} t-\frac{\bar{A} t^{2}}{2}\right)-\lambda_{1} \iint(H-\bar{H})\right)$,

where $\dot{R}_{2}$ is given by (21). It can thus be shown that a natural expression for $u_{3}$ is (22). 


\section{Numerical results}

We discretize the shallow water equations with the semi-implicit Preissman scheme (see [9] or [5]). When discretizing, it is possible to choose Preissman coefficient $\theta$ and Courant number $C_{r}$ (namely $\theta=0.5$ and $C_{r}=1$ ) such that the discretization does not introduce numerical damping for the linear equations. However, with this choice of parameters, the numerical errors are not damped and the solution obtained becomes non-smooth. Therefore we use a $\theta>0.5$ even if it generates an artificial stabilization due to the numeric damping. To overcome this difficulty, we compare the stabilization rate of open-loop to closed-loop systems.

\subsection{Simulation with a complete stabilization}

In this section, we set the Preissmann coefficient $\theta$ to the value 0.51 and the time-step $\Delta t=0.2$ and the space-step $\Delta x=0.5$. We consider the following initial conditions $\widetilde{D}=0, \widetilde{S}=0$ and for all $x$ in $[0, L], \widetilde{H}(x)=0.02 x+0.88, \widetilde{V}(x)=\sin ^{2}\left(\frac{x \pi}{L}\right)$. Let us study the stabilization problem of the fluid and note that we want the tank of length $L=12$ to stay the most close as possible from its initial position.

Let us compare the three following control laws: the null control, $u=0$, the control (20) given by Theorem 2 and the control (22) given by Theorem 3, with the gains $\lambda_{1}=0.01, \lambda_{2}=0.05$ and $\lambda_{3}=0.04$. We note in Figures 2 and 3 that the controls defined in Sections 3.2 and 3.3 succeed in stabilizing the fluid's state contrary to the system without control, where some oscillations of the fluid stay even after 100 seconds. In Figure 4, we check that the control of Section 3.2 stabilize the tank's speed around the value 0 . We note that with this controller, the tank's position tends to a constant $(\approx-0.05)$. This motives to use a forward approach as in Section 3.3 to track this value to 0. This control realize the complete stabilization of the tank. Note that at the bottom of Figure 4, we have the plot of the accelerations, therefore we have the controls.

\subsection{Importance of the non-linear terms of the shallow water equations}

In this section we consider the following equilibrium: $\bar{D}=0, \bar{S}=0, \bar{A}=0$, $\bar{H}=1.5$ and $\bar{V}=0$. Note that the shallow water equations linearized around this equilibrium are uncontrollable, even locally (see [7]). Indeed the function $H, V$ : $[0,+\infty) \times[0, L] \rightarrow \mathbb{R}$ and $D:[0,+\infty) \rightarrow \mathbb{R}$ defined by, for all $t \geq 0$ and for all $x$ in $[0, L]$

$$
\begin{gathered}
D(t)=0, \quad H(t, x)=1+\sin ^{2}\left(\frac{\pi x}{L}\right), \\
V(t, x)=-2 \frac{\pi}{g L} t \cos \left(\frac{\pi x}{L}\right) \sin \left(\frac{\pi x}{L}\right),
\end{gathered}
$$

are solutions of the linearized equations with $u=0$. However the nonlinear shallow water equations are locally controllable (see [1]), we expect (and we check) numerically that the nonlinear equations are stabilizable. 

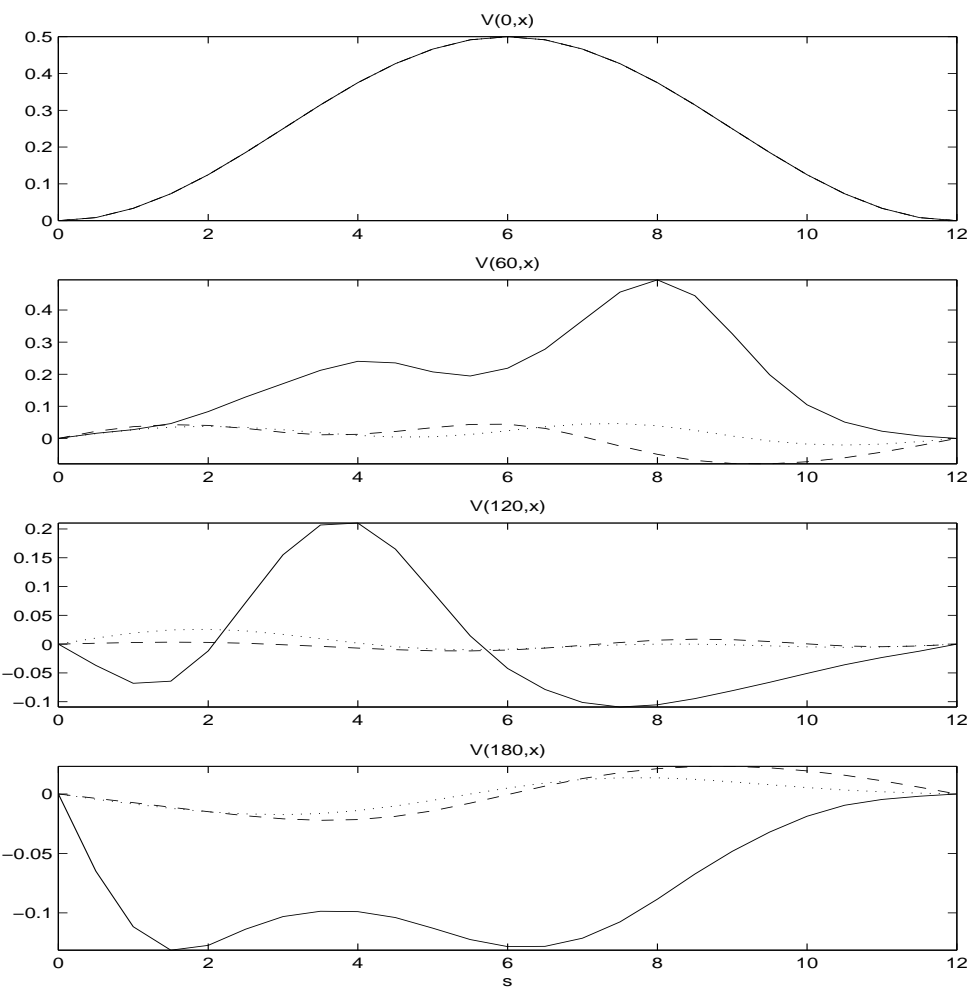

Figure 2: Fluid's speed in the tank at time $t=0, t=60, t=120$ and $t=180$ seconds. The curve - is the fluid with the null control, - - with the control (20) of Section 3.2 and $\cdots$ with the control (22) of Section 3.3.

To do this, we consider as initial condition the value of the functions (23)-(24) at $t=0$ and we implement the feedback of Section 3.3. More precisely let us define $L=7.5$ and the following initial conditions, $\widetilde{D}=0, \widetilde{S}=0$ and, for all $x$ in $[0, L]$, $\widetilde{H}(x)=1+\sin ^{2}\left(\frac{\pi x}{L}\right), \widetilde{V}(x)=0$. We set $\lambda_{1}=0.4, \lambda_{2}=0.1$ and $\lambda_{3}=0.1$. We choose $\theta=0.5001$ which is very close to the critical value (namely 0.5 ). Therefore we have non-smooth numerical solutions (see Figure 6). We observe in Figure 7 that the tank stays very close to the initial position but succeed in stabilizing the fluid's speed (see Figure 5) and the fluid's height (see Figure 6).

\section{Conclusion}

In this paper we have computed a class of feedback which numerically stabilizes the system fluid-tank. A further work of this paper is to prove that the stabilization is achived. One possible way is described by Remark 3.1.

In this paper the control laws are feedbacks of all variables of the system fluidtank. But to implement these feedbacks and to use it for a physical application it is important to look for a feedback depending only of variables which are easy to compute. Thus it is important to explicit a stabilizing output-feedback where the output is the height of the fluid at the boundary of the tank, the time and the 

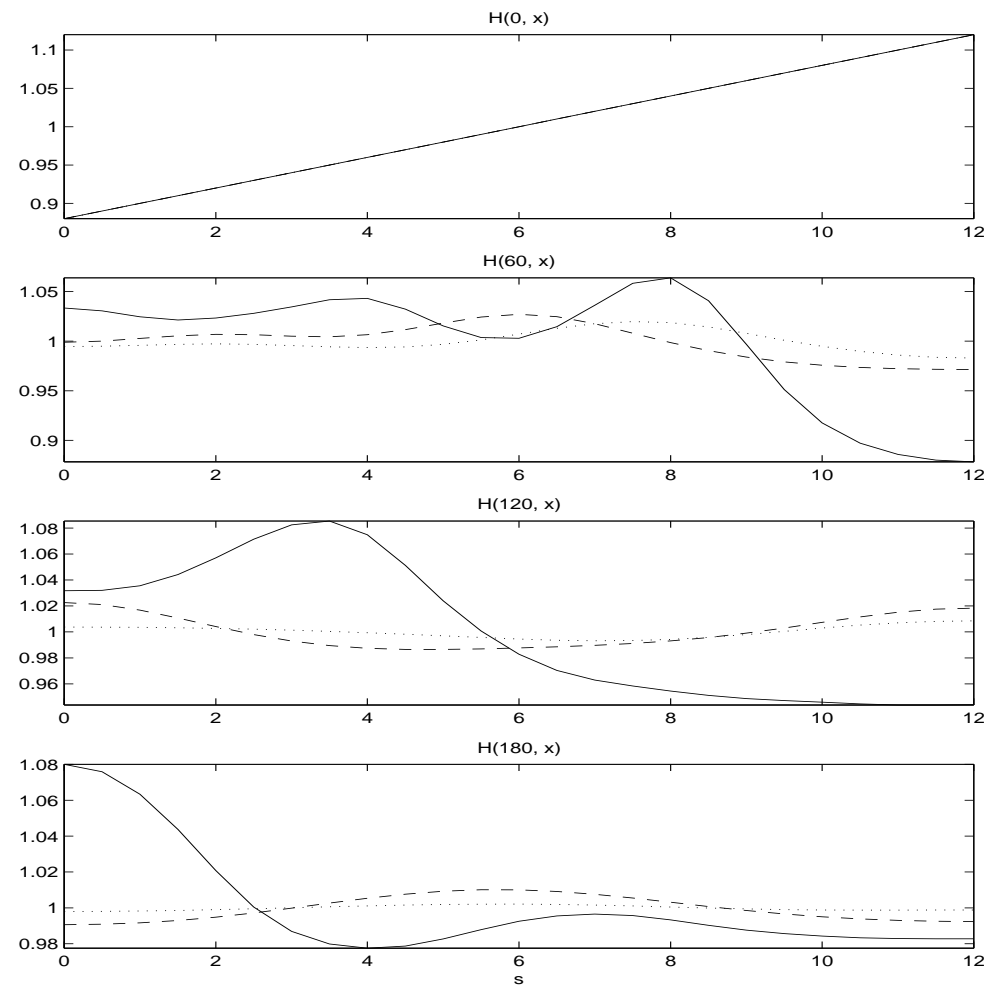

Figure 3: Fluid's height in the tank at time $t=0, t=60, t=120$ and $t=180$ seconds. The curve - is the fluid with the null control, - - with the control (20) of Section 3.2 and $\cdots$ with the control (22) of Section 3.3.

trajectory of the tank only. An other further work is to study a Lyapunov approach to find such feedbacks.

\section{References}

[1] Coron J.M. (2001). Local controllability of a 1-D tank containing a fluid modeled by the shallow water equations. Preprint, Université Paris-Sud, number 2001-28.

[2] Coron J.M., B. d'Andréa Novel and G. Bastin (1999). A lyapunov approach to control irrigation canals modeled by saint-venant equations. European Control Conference. Karlsruhe, Germany.

[3] Debnath L. (1994). Nonlinear water waves, Academic Press, Boston.

[4] J.-F. Gerbeau et B. Perthame, Derivation of viscous Saint-Venant system for laminar shallow water; numerical validation, rapport de recherche numéro RR4084, INRIA Rocquencourt, 2000.

[5] Graf W.H. (1998). Fluvial Hydraulics. John Wiley \& Sons. 

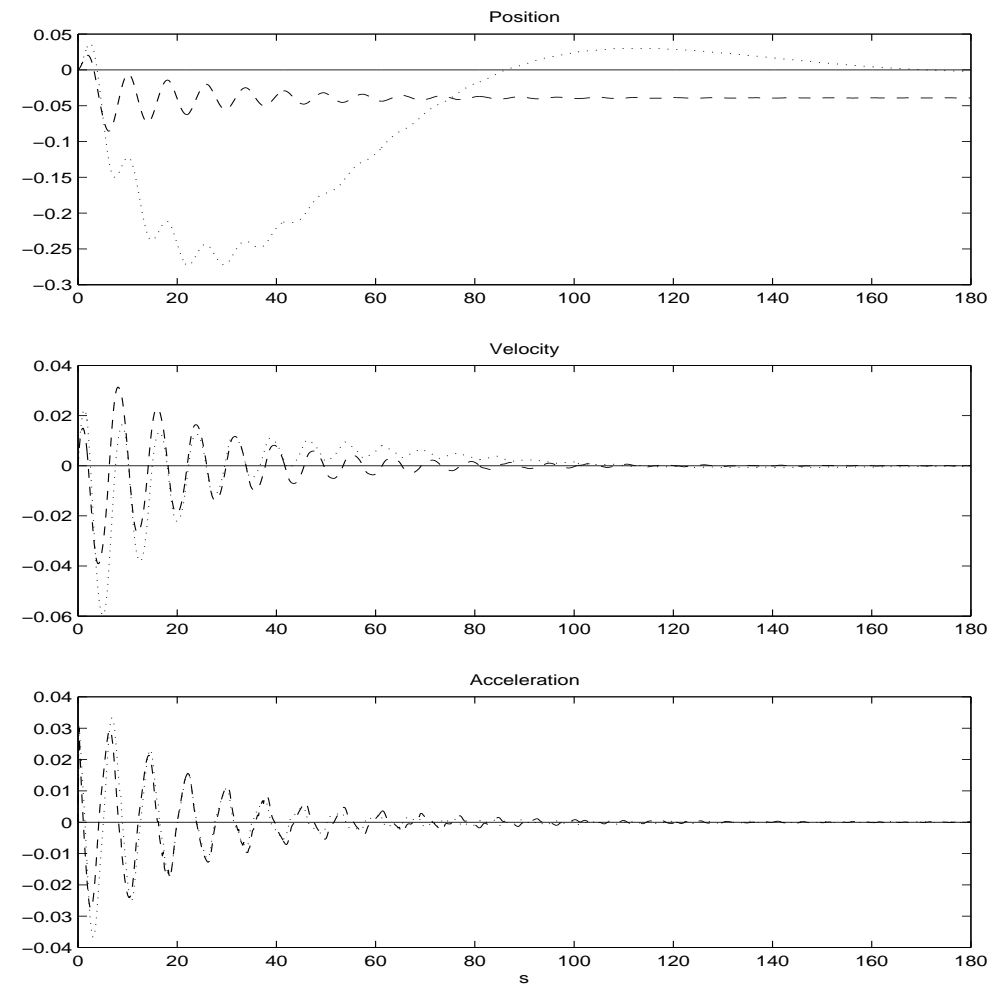

Figure 4: Trajectory of the tank in closed-loop with the null control (-), with the (20) control of Section $3.2(--)$ and with the control (22) of Section $3.3(\cdots)$. At the top, we have the plot of the position, in the middle, the velocity and at the bottom, the acceleration in function of the time. 

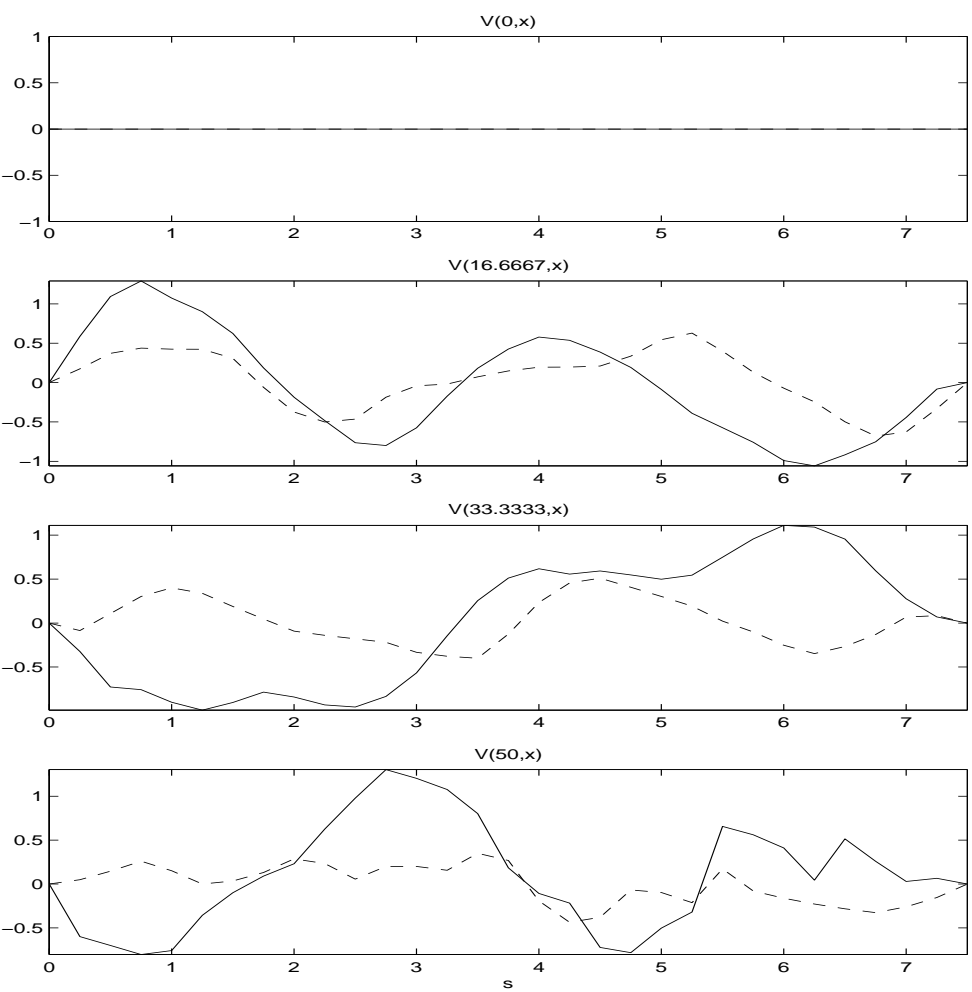

Figure 5: Fluid's speed in the tank at time $t=0, t=16.6, t=33.3$ and $t=50$ seconds. The curve - is the fluid with the null control, - - with the control of Section 3.3.

[6] M. Grundelius, Iterative optimal control of liquid slosh in an industrial packaging machine, Conference on Decision and Control, Sydney, Australie, 2000.

[7] Dubois F., N. Petit and P. Rouchon (1999).Motion planning and nonlinear simulations for a tank containing a fluid. European Control Conference. Karlsruhe, Germany.

[8] Li Ta-tsien and Wen-ci Yu (1995). Boundary value problems for quasilinear hyperbolic systems. Duke University, Durham.

[9] Malaterre P.O. (1994). Modelisation, Analysis and LQR Optimal Control of an Irrigation Canal. PhD thesis, LAAS-CNRS-ENGREF-Cemagref, France.

[10] Mazenc F. and L. Praly (1996). Adding integrations, saturated controls, and stabilization for feedforward systems. IEEE Trans. Automat. Control, 41 (11), $1559-1578$.

[11] Mottelet S. (2000). Controllability and stabilization of a canal with wave generators. SIAM J. Control Optimization, 38 (3), 711-735.

[12] Mottelet S. (2000). Controllability and stabilizability of liquid vibration in a container during transportation. Conference on Decision and Control. Sydney, Australy. 

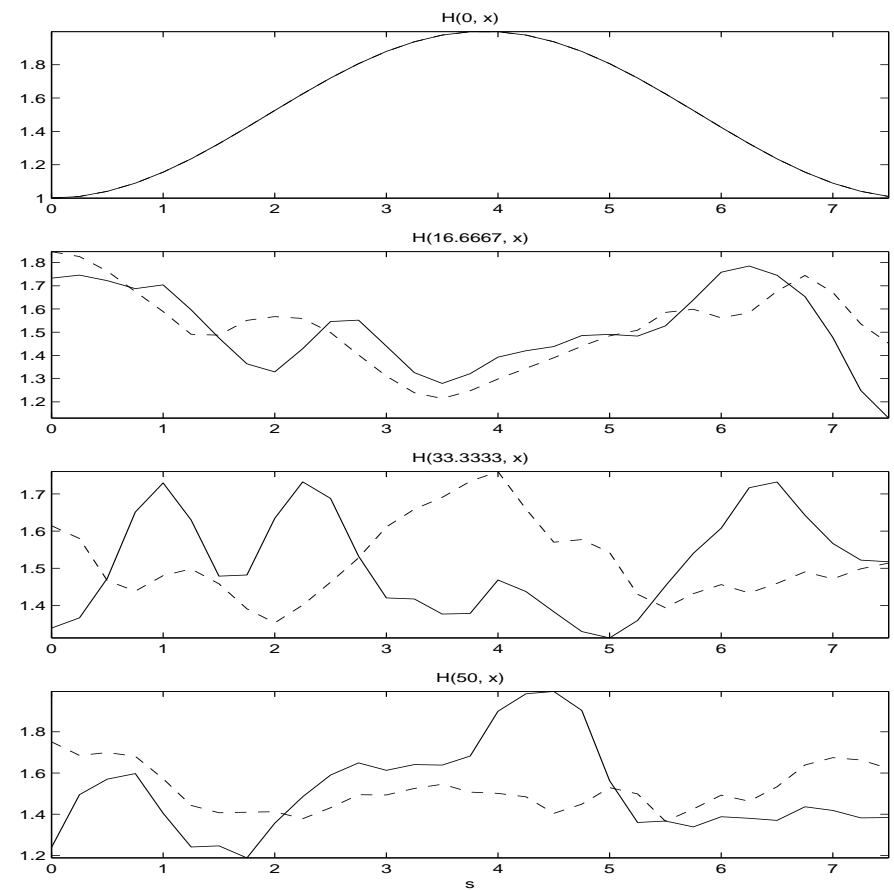

Figure 6: Fluid's height in the tank at time $t=0, t=16.6, t=33.3$ and $t=50$ seconds. The curve - is the fluid with the null control, - - with the control of Section 3.3.

[13] Petit N. and P. Rouchon (2000). Dynamics and solutions to some control problems for water-tank systems. CIT-CDC, (00-004).

[14] Prieur C., Diverses méthodes pour des problèmes de stabilisation, thèse, université Paris-Sud, 2001.

[15] Saint-Venant B. de (1871). Théorie du mouvement non-permanent des eaux avec applications aux crues des rivières et à l'introduction des marées dans leur lit, Comptes-rendus de l'académie des Sciences, Paris, 73, 148-154, 237-240.

[16] Serre D. (1996). Systèmes de lois de conservations I and II. Diderot éditeur, Art et Sciences.

LABORATOIRE D'ANALYSE NUMÉRIQUE ET EDP, UNIVERSITÉ PARIS-SUD,

BÂTIMENT 425,

91405 Orsay, France

Christophe.Prieur@math.u-psud.fr

CESAME,

Université Catholique de Louvain, 4, Av. Georges Lemaitre,

B-1348 Louvain-LA-Neuve, Belgium

dehalleux@csam.ucl.ac.be 

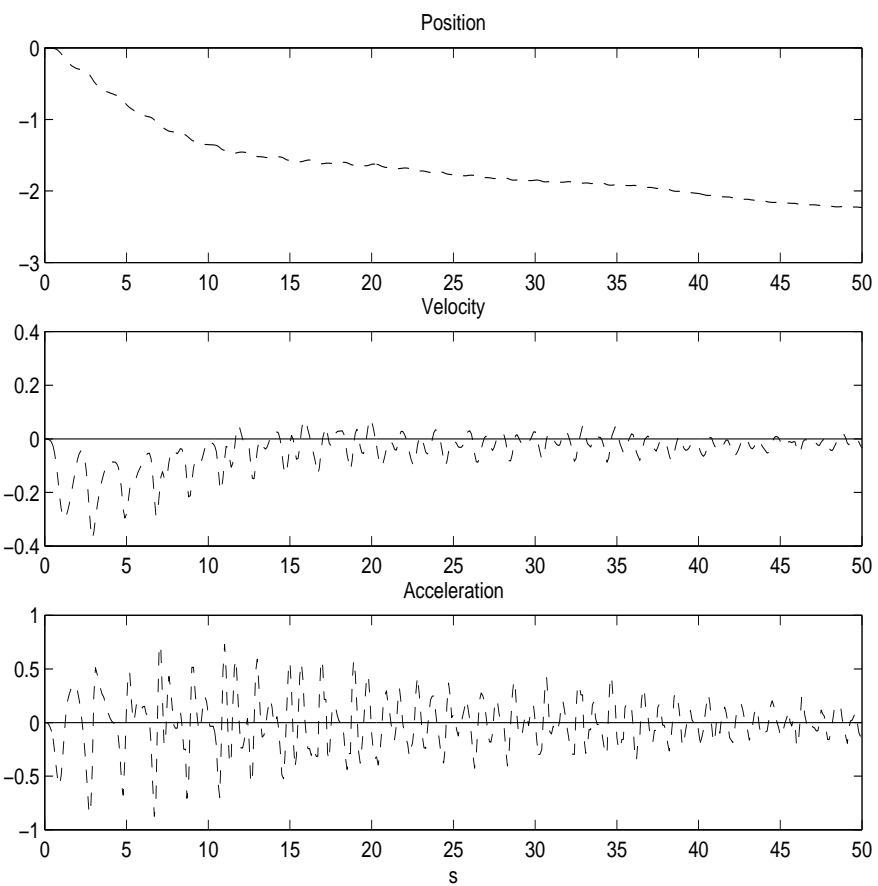

Figure 7: Trajectory of the tank in closed-loop with the null control $(-)$, with the control of Section $3.3(--)$. At the top, we have the plot of the position, in the middle, the velocity and at the bottom, the acceleration in function of the time. 